\title{
South African boys in New Zealand: Immigrants' retrospective accounts of adapting to life in a new country during adolescence
}

\section{Teswell White}

\begin{abstract}
It is now estimated that around 50 million displaced persons (highest number since the end of World War 2), and as many as 10 million are 'on the move' between countries at the present time. Mass migration is now undoubtedly be one of the biggest issues demanding global attention. A major concern for the United Nations over the next few years will certainly be how to deal with mass migration. The dominant messages from the mass media and politicians are dominated by 'political correctness', but fail to focus on the main issue of how newcomers and their children will function in their new location after arrival. Whilst the discourses of assimilation are becoming more pronounced, the question might then justifiably be asked, "What happens to the children of newcomers after arrival?' This is a critical question in every sense and raises the issue of immigrant youth adaptation and experiences of identity and place in this age of shifting demographics, large-scale migration, and multicultural communities.
\end{abstract}

Not long ago most of the people who came to New Zealand were coming from parts of the United Kingdom with relatively few coming from anywhere else. Relatively little is known about the psychosocial adaptations and self-identities of young immigrants coming of age in the compound multi-ethnicity of contemporary New Zealand. Therefore, the overall aim of this study was to better understand the complexities of contemporary youth adaptation, at a time when immigrants into New Zealand are coming from an increasingly diverse range of nations. Specifically, the aim was to offer insights into how personal, social and cultural factors might combine to shape male adolescent experiences, and how these insights may be of use to teachers who work with this age group in culturally and ethnically diverse contexts.

I used detailed one-to-one interviews and drew on a social constructionist theoretical framework, to map the complex ways in which the boys said they negotiated, navigated, contested and resisted their engagements with the different discourses and stories about them that operate in their high school environment. Much of the analysis included an examination of how they reconstructed adolescence from a position of young adulthood. The sort of data that I collected is not designed to offer a logical set of generalized statements about the immigration. Rather, the individualised comments offer a glimpse into the complexity of the individual experience of adapting to life in a new country for South African boys.

The findings of this study tend to suggest that there is a lot more going on in the lives of immigrant boys than what essentialist categories assume. 'Identity uncertainty and confusion and surveillance and policing of school masculinities' were key themes raised during the analysis of the interviews. A main argument in the study is that cultural and ethnic identities for South African boys were defined

Wilf Malcolm Institute of Educational Research, Faculty of Education, University of Waikato, Hamilton, New Zealand ISSN: 2382-0373

Permanent Research Commons link to full thesis: http://hdl.handle.net/10289/10746 (pp. 123-124) 
through the possibilities offered to them in their social contexts. The themes draw attention to a distinctive contradiction between cultural messages from home to 'remain rooted' in their heritage culture versus messages from the host to either 'fit in' or to be socially excluded. This home/host contradiction had different meanings for individual boys, and this varied across the different ethnic groups.

The findings also tend to suggest that the masculine identities that South African boys constructed and lived out were not fixed and unproblematic but rather fluid and in constant flux. They show the role of schooling in the shaping of a range of different male heterosexual subjectivities, and how the subject positions that the boys inhabited held different configurations of threat and promise of social inclusion based on their particular South Africanness. Although these findings are not representative of all South African boys in New Zealand, they do suggest some of the ways that immigration and masculinity might intersect during adolescence and the ways aspects of masculine identity expressions come to reflect the social relations at school. 